resinas compostas diretas do mesmo fabricante. Materiais e métodos: Amostras uniforrmes de três blocos de resina composta para CAD/CAM [Tetric CAD (TC), Grandio blocs (GB) e Brilliant Crios (BC)] e de quatro resinas compostas diretas [Tetric EvoCeram (TEC), GrandioSO (GS), Brilliant EverGlow Translucent e Universal Shade (BET) e (BEU)] foram submetidas ao teste de flexão a 3 pontos a uma velocidade de 1 $\mathrm{mm} / \mathrm{min}$ ( $\mathrm{n}=10$ a 15 por grupo). Posteriormente, duas amostras de cada grupo foram submetidas a ensaios de microdureza de Vickers, sendo feitas 5 indentações em cada $(n=10$ por grupo). Os dados resultantes da resistência à flexão foram analisados usando o teste One-Way ANOVA considerando a correção de Bonferroni para as análises post-hoc $(0,05)$. Os resultados do módulo de flexão e a microdureza de Vikers foram analisados usando o teste Welch's ANOVA consideranto a correção de Games-Howell para as análises post- hoc $(0,05)$. Resultados: A resistência à flexão e o modulo de flexão alcançaram valores médios que variam de 81,1 $\mathrm{MPa}$ (BEU) a 246,5 MPa (GB) e de 10,6 GPa (BEU) a 20,3 GPa (GB), respetivamente. BET (49,9 HV) e BEU (50,9 HV) foram os grupos com menores valores médios de microdureza e os grupos GS (121,2 HV) e GB (136,2 HV) tiveram os valores médios mais elevados. As anásises post-hoc mostraram diferenças estatisticamente significativas na resistência à flexão, módulo de flexão e microdureza de vickers ao comparar os BRC (BC, GB e TC) às resinas compostas diretas, à excepção da resina composta direta GS quando comparada ao bloco GB para a microdureza. Conclusões: Dentro das limitações deste estudo, foram observadas diferenças entre os BRC e as resinas compostas diretas na resistência à flexão, no módulo de flexão e microdureza para o protocolo realizado. O nosso estudo sugere assim que a generalidade dos BRC tem melhores propriedades mecânicas que as resinas compostas diretas. Dentro de cada categoria, blocos de resina ou resinas compostas diretas, o bloco Grandioblocs e a resina Grandioso são os materiais com as melhores propriedades mecânicas testadas.

http://doi.org/10.24873/j.rpemd.2019.12.574

\#113 A estabilidade da cor dos bulk fill pode ser influenciada pelo sistema adesivo?

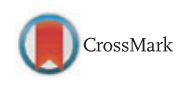

Ana Sofia Leitão Belchior*, Carlos Ferreira de Almeida, Cláudia Alexandra da Silva Rodrigues, André Correia, Rita Noites

Universidade Católica Portuguesa

Objetivos: Avaliar a influência de diferentes sistemas adesivos na alteração colorimétrica de duas resinas bulk fill. Materiais e métodos: Neste estudo experimental in-vitro, foram elaborados 270 discos com a resina composta UD3 (Micerium) que foram divididos em 9 grupos, um de controlo, sem adesivo, e 8 grupos de estudo nos quais foram aplicados: Excite F (Ivoclar Vivadent), Scotchbond Universal (3M), Clearfil SE Bond 2 (Kuraray), Futurabond NR (VOCO), OptiBond XTR (Kerr), Clearfil Universal Bond (Kuraray), Futurabond U (VOCO) e One Coat 7 Universal (Colténe). Foram elaborados 540 discos de cada resina: Fill-Up (Colténe) e Xtra-Fill (VOCO) sobre os discos iniciais. A avaliação colorimétrica foi realizada inicialmente, 15 e
30 dias com recurso a um Espectrofotómetro (VITA Easyshade ${ }^{\circledR}$ Compact). Durante este período os discos foram submetidos a uma solução corante (café e coca-cola). Foi feita a análise estatística utilizando o SPSS $^{\circledR}$ v.25.0, com recurso a testes paramétricos (Anova one way, pos-hoc Bonferroni) e não paramétricos (Kruskal Wallis e U-Mann Whitney) em que se considerou estatisticamente significativo um $p \leq 0,05$. Resultados: Observaram-se diferenças significativas entre os grupos após os 15 e 30 dias de pigmentação, com um diminuição da luminosidade e aumento de cor amarelo/castanha. Os adesivos self-etch estudados apresentam menor variação global de cor, comparados com os universais, sendo o total-etch o que apresenta valores mais significativos. $\mathrm{O}$ adesivo que apresentou menor variação global média de cor foi o Clearfil SE Bond 2, contudo, o que apresentou menor variação de resultados foi o Futurabond NR. O adesivo universal com menor variação foi o Clearfil Universal Bond. Verificou-se que a Xtra-Fill é menos suscetível a alterações de cor ao contrário da outra resina estudada. Conclusões: O sistema adesivo utilizado poderá ter impacto na estética da restauração a longo prazo. Aquando da utilização de resinas translúcidas como é o caso das resinas bulk fill o sistema adesivo a utilizar poderá ter impacto. Mais estudos são necessários.

http://doi.org/10.24873/j.rpemd.2019.12.575

\#114 Comparação da mensuração de cor visual, espectrofotométrica e pela fotografia polarizada

Ricardo Jorge Lourenço Rodrigues*, Cristiano Gabriel Azevedo Pereira Teixeira Alves, Francisco José Santiago Fernandes Amado Caramelo, João Paulo Tondela

Faculdade de Medicina da Universidade de Coimbra

Objetivos: A mensuração de cor em medicina dentária pode ser realizada tanto pelo método visual como por métodos instrumentais. A fotografia polarizada mostra-se como um método emergente para mensuração e comunicação da cor dentária com o laboratório; O objetivo deste estudo é comparar a precisão dos métodos visual, espectrofotométrico e pela fotografia polarizada na mensuração de cor. Materiais e métodos: Uma amostra de 33 discos de resina composta com cores aleatórias foi submetida à mensuração de cor visual por comparação com a escala VITAPAN Classical ${ }^{\circledR}$ por 10 médicos dentistas pós graduados em reabilitação oral protética e/ou dentisteria operatória sob condições padronizadas. A amostra e a escala VITAPAN Classical ${ }^{\circledR}$ foram medidas com o espectrofotómetro clínico Vita Easyshade ${ }^{\circledR} \mathrm{V}$ e fotografadas com polarização cruzada. Foi feita a extrapolação das cores escolhidas pelos observadores com base nas cores medidas pelos dois métodos instrumentais em CIE L*a*b*. Para as três coordenadas CIE L*a*b* obtidas pelos três métodos foram analisadas a correlação de Pearson, correlação intraclasse (ICC) e contruídos gráficos Bland Altman. Foi também calculada a média de $\Delta \mathrm{E}$ entre os três métodos e o $\Delta \mathrm{E}$ máximo para $95 \%$ segundos os limites de concordância dos gráficos Bland Altman. Resultados: Foram obtidos valores estatisticamente significativos de ICC e correlação de Pearson $(p<0,001)$ para todos os pares comparados para todas as coordenadas CIE L*a*b*. A mensu- 
ração espectrofotométrica e a análise da fotografia polarizada mostram correlação forte para $L^{*}(r=0.99)$ e $a^{*}(r=0,94)$ e moderados para $b^{*}(r=0,538)$. Quando comparados com a mensuração de cor visual, foram obtidos maiores valores de ICC para a fotografia polarizada para as coordenadas $a^{*}(0.83)$ e $b^{*}(0.83)$ e maior valor de ICC para o espectrofotómetro para a coordenada $L^{*}(0.80)$. Foi obtido $\Delta$ E médio de 6.72 para o par fotografia-observadores, 5.93 para o par espectrofotómetro-observadores e 3.29 para o par fotografia-espectrofotómetro. Os $\Delta \mathrm{E}$ máximos calculado segundo os limites de concordância dos gráficos Bland-Altman das três coordenadas CIE L*a*b* foram 14.27 para o par espectrofotómetro-observadores, 12,45 para o par fotografia-observadores e 7.39 para o par fotografia-espectrofotómetro. Conclusões: Tendo em conta as limitações deste estudo, a fotografia polarizada mostrou precisão semelhante ao espectrofotómetro considerando o limite de percetibilidade clínica. A mensuração visual mostrou diferenças clinicamente percetíveis em relação aos dois métodos instrumentais

http://doi.org/10.24873/j.rpemd.2019.12.576

\#115 Prevalência de lesões periapicais e associação com o comprimento da obturação canalar

João Meirinhos*, Ana Beatriz de Cristino Roque Jordão Pereira, Baruwa Abayomi, Jorge Martins, António Ginjeira

Faculdade de Medicina Dentária da Universidade de Lisboa

Objetivos: O objetivo deste estudo foi analisar in vivo a prevalência de lesões periapicais e a sua associação com comprimento da obturação canalar, através da análise de exames de tomografia computorizada de feixe cónico (СВCT). Materiais e métodos: 5 observadores pré-calibrados, realizaram uma análise de 1160 exames de CBCT referentes a pacientes diferentes em 8 clínicas dentárias. De modo a uniformizar as características dos exames, foi estabelecido como parte dos critérios de inclusão que apenas volumes de arcada completa e tamanhos de voxel igual ou inferior a $200 \mu \mathrm{m}$ seriam aceites. Foram incluídos 20,836 dentes, sendo que 2,305 apresentavam tratamento endodôntico. Cada dente foi classificado de acordo com o comprimento da obturação canalar ('curto' quando a porção mais apical da obturação está a mais de $2 \mathrm{~mm}$ de distância do ápex radiográfico; "correto" quando a obturação de encontra entre 0 e $2 \mathrm{~mm}$ do ápex radiográfico; e "sobre-obturação" quando está para além do ápex radiográfico). Tendo como base uma amostra inicial de 319 dentes avaliados duas vezes com 1 mês de intervalo, foram calculadas a coerência intra e inter-observador, tendo os valores sido acima dos 0.61 . O z-test foi utilizado para comparar as proporções entre diferentes sub-grupos e o odds ratio foi calculado para determinar a associação entre o comprimento da obturação e lesões periapicais. Resultados: A prevalência de periodontite apical em dentes com tratamento endodôntico com uma obturação aquém do ápex foi de $72.7 \%$, sendo 3.1 vezes maior a possibilidade de existirem lesões em comprimentos de obturação 'curto' quando comparado com "correto". Por outro lado, as proporções de "correto" (46.0\%) e "sobre-obturação" (45.4\%) não demonstraram diferenças significativas entre si. Dentes maxilares apresentaram, também, uma prevalência de lesões periapicais superior. Conclusões: Os resultados expressam uma elevada prevalência de lesões periapicais em dentes que apresentam uma obturação aquém do ápex radiográfico. Controlos radiográficos deverão ser realizados de forma a controlar lesões apicais em dentes com tratamentos endodônticos previamente realizados, possível aparecimento de novas e consequentemente necessidade de abordagem de possíveis futuros fracassos endodônticos.

http://doi.org/10.24873/j.rpemd.2019.12.577

\section{\#116 Prevalência de lesões periapicais no segundo molar maxilar com ou sem raízes fundidas}

Beatriz Pereira*, João Meirinhos, Abayomi O. Baruwa, José Gouveia, Jorge N. R. Martins, António Ginjeira

\section{FMDUL}

Objetivos: O objetivo deste estudo foi a determinação da prevalência de lesões periapicais em segundos molares maxilares com raízes fundidas apresentando tratamento endodôntico, com recurso a imagens de tomografia computorizada de feixe cónico (CBCT) em condições in vivo. Materiais e métodos: Um total de 1.160 CBCT's, referentes a diferentes pacientes, foram analisados em várias clínicas localizadas nas localidades do Porto, Aveiro, Lisboa, Moita e Setúbal durante um período de 12 meses compreendido entre Janeiro e Dezembro de 2018. Foi pré-definido como parte dos critérios de inclusão do presente trabalho que seriam apenas aceites volumes de arcada completa e com tamanhos de voxel iguais ou abaixo de $200 \mu \mathrm{m}$. Os testes de Cohen kappa e ICC foram realizados para a obtenção da concordância intra e inter-observadores, respectivamente, tendo como base a avaliação inicial de uma amostra de 319 dentes avaliados duas vezes com um intervalo de 30 dias entre avaliações. Ambos os testes apresentaram resultados acima dos 0.61 . Como amostra final, obtiveram-se imagens de 20.836 dentes dos quais 1.346 eram segundos molares maxilares. O desfecho primário foi a obtenção da prevalência de lesões periapicais nos segundos molares maxilares com ou sem fusão radicular. As proporções são expressas num intervalo de confiança de $95 \%$. O Z-test para proporções foi utilizado para comparar as proporções entre sub-grupos e o odds ratio foi calculado para compreender a associação entre as lesões e o tipo de configuração radicular. Resultados: A prevalência de lesões periapicais em dentes com fusão radicular e tratamento endodôntico foi de $73.6 \%$ (61.7\%-85.5\% CI 95\%), enquanto que a proporção de lesões em dentes sem fusão radicular e tratamento endodôntico foi de 64.7\% (55.4\%-73.9\% CI 95\%). Esta diferença não foi considerada estatisticamente significativa ( $p>0.05)$. Os dentes com raízes fundidas e tratamento endodôntico apresentaram 1.3 vezes maior probabilidade de estarem associados a presença de lesões periapicais do que dentes com tratamento endodôntico sem fusão radicular. Conclusões: Há uma tendência para uma maior proporção de lesões periapicais em dentes com tratamento endodônticos que apresentam fusão radicular quando com- 